Supporting Information

\title{
Selective production of phenolic monomers and biochar by pyrolysis of lignin with internal recycling of heavy oil
}

Tianlong Liu, ${ }^{\dagger}$ Asuka Mori, ${ }^{\dagger}$ Ryohei Arai,${ }^{\dagger}$ Shusaku Asano,,${ }^{\dagger} \pitchfork$ Shinji Kudo,${ }^{\dagger \dagger}$ and Junichiro Hayashi $*, \dagger, \$$

$\dagger$ Interdisciplinary Granduate School of Engineering Sciences, Kyushu University, 6-1, Kasuga Koen, Kasuga 816-8580, Japan

$\$$ Institute for Materials Chemistry and Engineering, Kyushu University, 6-1, Kasuga Koen, Kasuga 816-8580, Japan

*junichiro_hayashi@cm.kyushu-u.ac.jp 


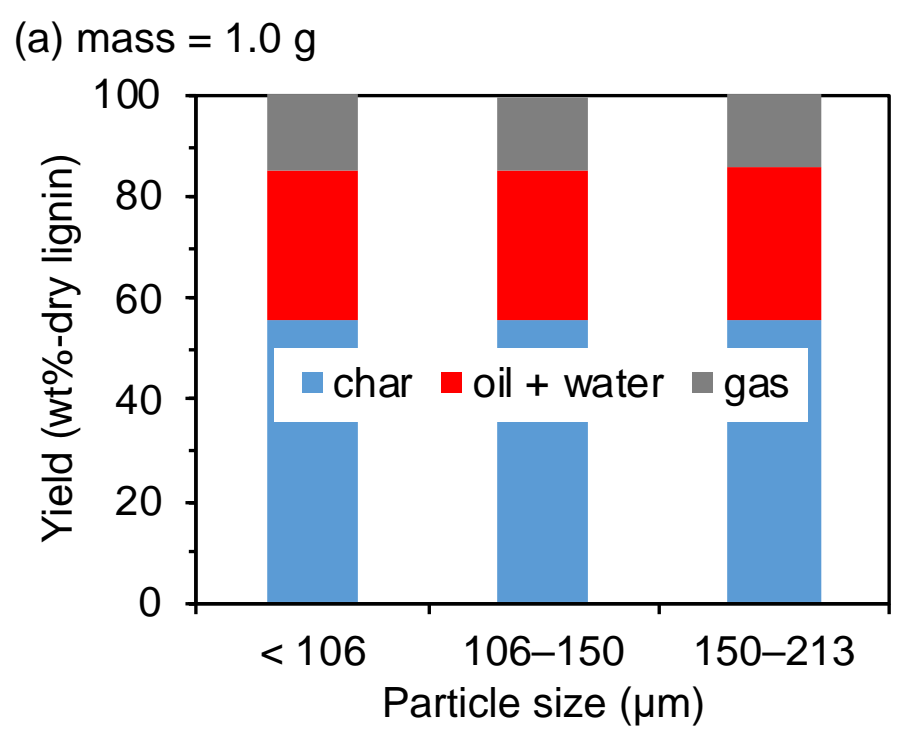

(b) particle size: $106-150 \mu \mathrm{m}$

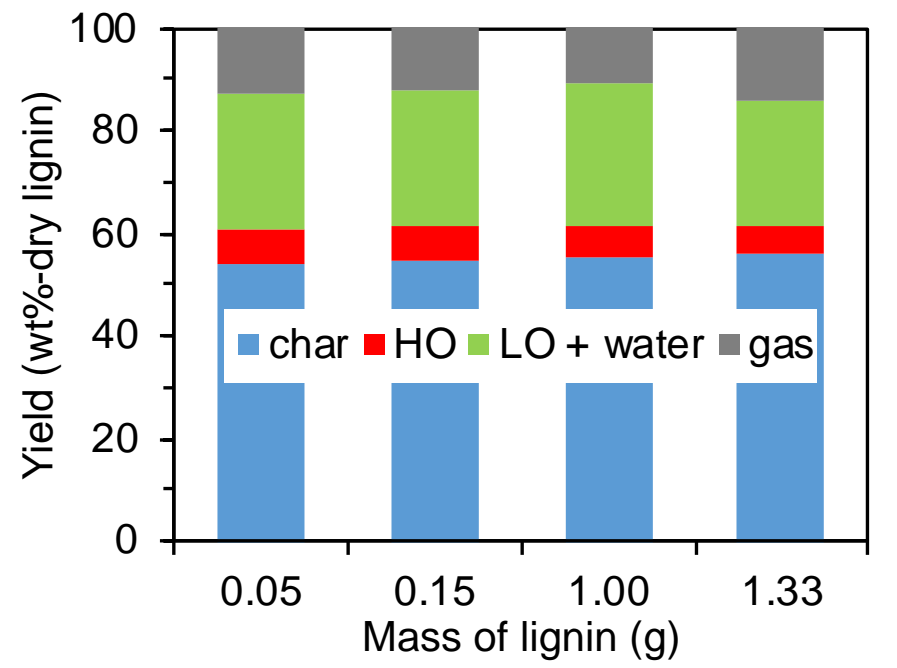

Figure S1. Yields of pyrolytic products as a function of (a) particle size and (b) mass of as-prepared lignin. 

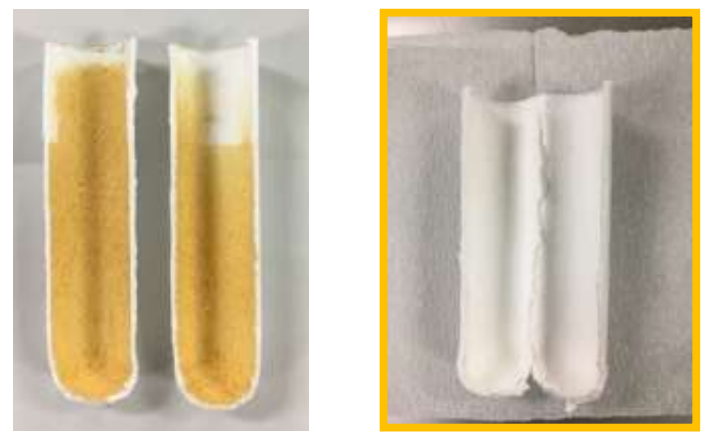

Figure S2. Photographs of spent thimble filters. Left: after a pyrolysis run with the parent lignin as the feedstock. In the run, the filter was set at the reactor downstream and heated at $130^{\circ} \mathrm{C}$. The brown colored solid was deposit of HO. Right: after another run with a fixed bed of the parent lignin between the reactor and the thimble filter. No color change occurred on the inner surface of the filter due to complete capture of $\mathrm{HO}$ by the lignin fixed bed. 


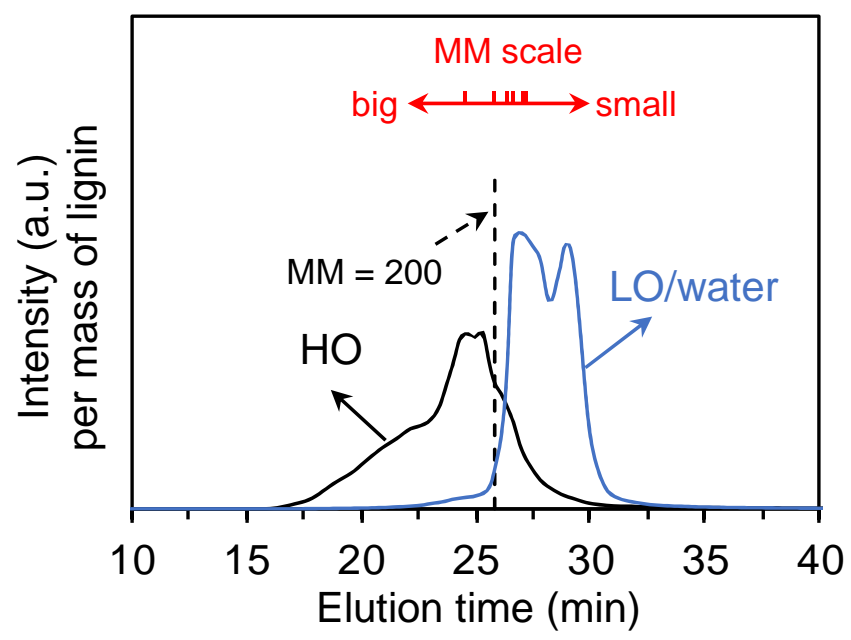

Figure. S3-1. GPC chromatograms LO/water and HO. The intensity was normalized to per mass of lignin. The $\mathrm{LO} /$ water from the run with the lignin fixed bed was recovered from the condensers by dissolution in tetrahydrofuran and then analyzed by GPC. The $\mathrm{HO}$ was from a run equivalent to the first run of nine runs in series. The $\mathrm{HO}$ was recovered from the thimble filter by dissolution in acetone, evaporative removal of acetone, redissolution in tetrahydrofuran, and then analyzed by GPC. Calibration between elution time and molecular mass was performed in reference to eight model compounds, as shown in the right table. It is seen that the major portion of LO had MM lower than 200. On the other hand, HO contains heavy components with MM far beyond 200. The eight MM scales correspond to the eight model compounds listed in the table below.

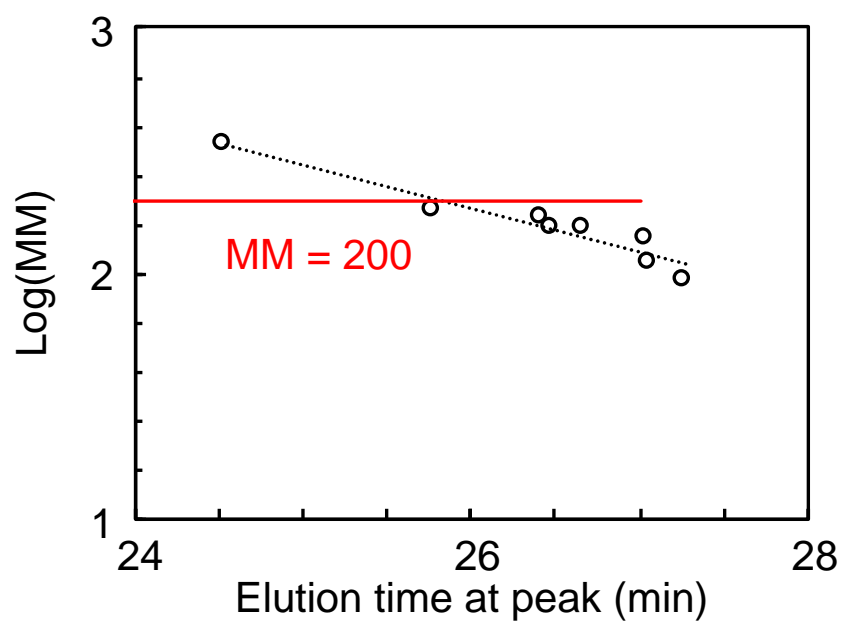

Figure. S3-2. Relationships between elution time and molecular mass (MM) of the eight model compounds: phenol (94), o-cresol (108), creosol (138), 4-ethylguaiacol (152), vanillin (152), acetovanillone (166), guaiacylacetone (180) and 1-(3,4dimethoxyphenyl)-2-(2-methoxyphenoxy)-1,3-propanediol (334). 


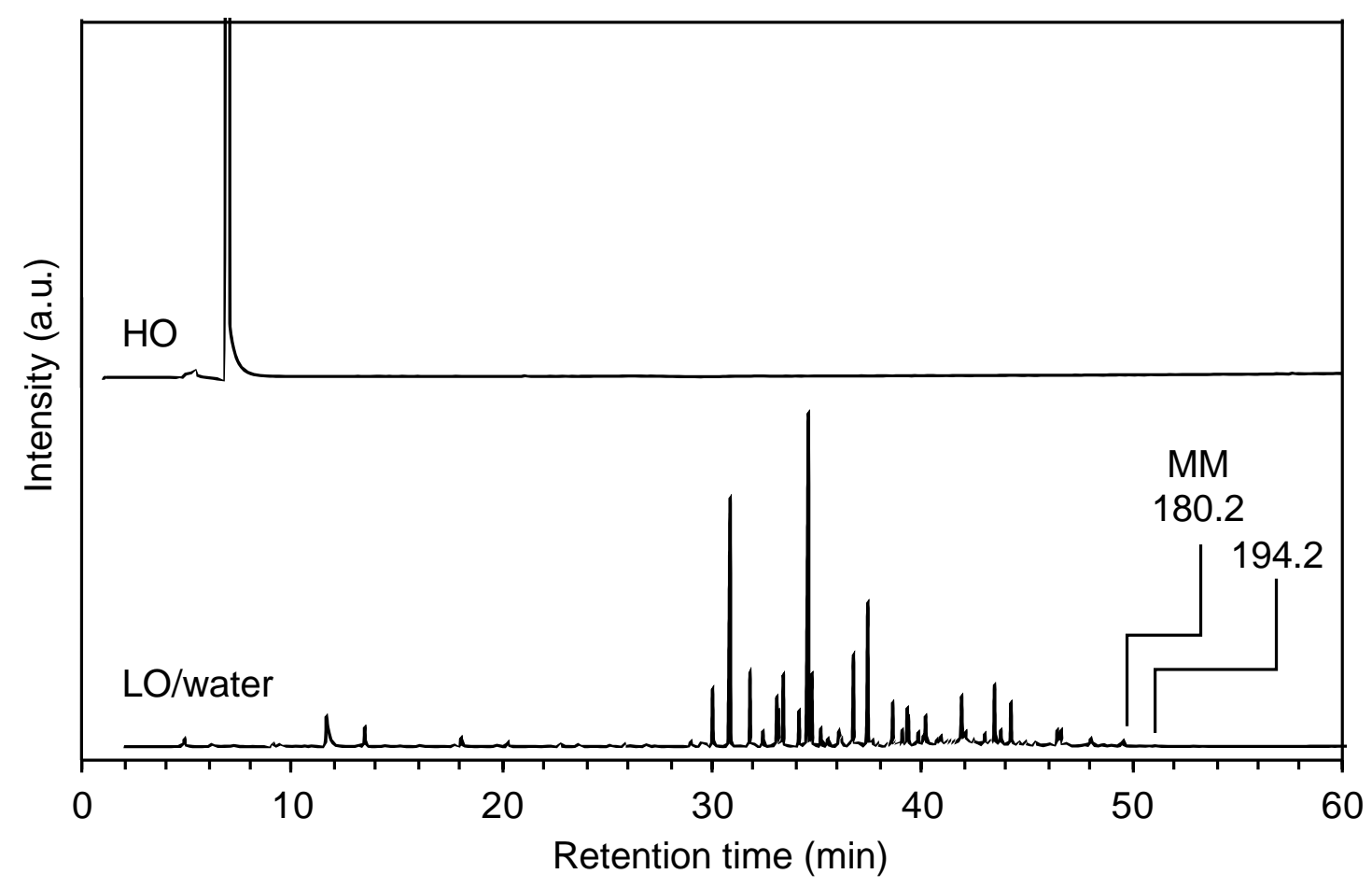

Figure S4. Typical GC/MS chromatograms of LO/water and HO. The LO/water was collected in the condensers from the run with the lignin fixed bed while the $\mathrm{HO}$ was from a run equivalent to the first run of nine runs in series. No peaks were found for HO. Only compounds at molecular mass $<200$ were detected in LO. 


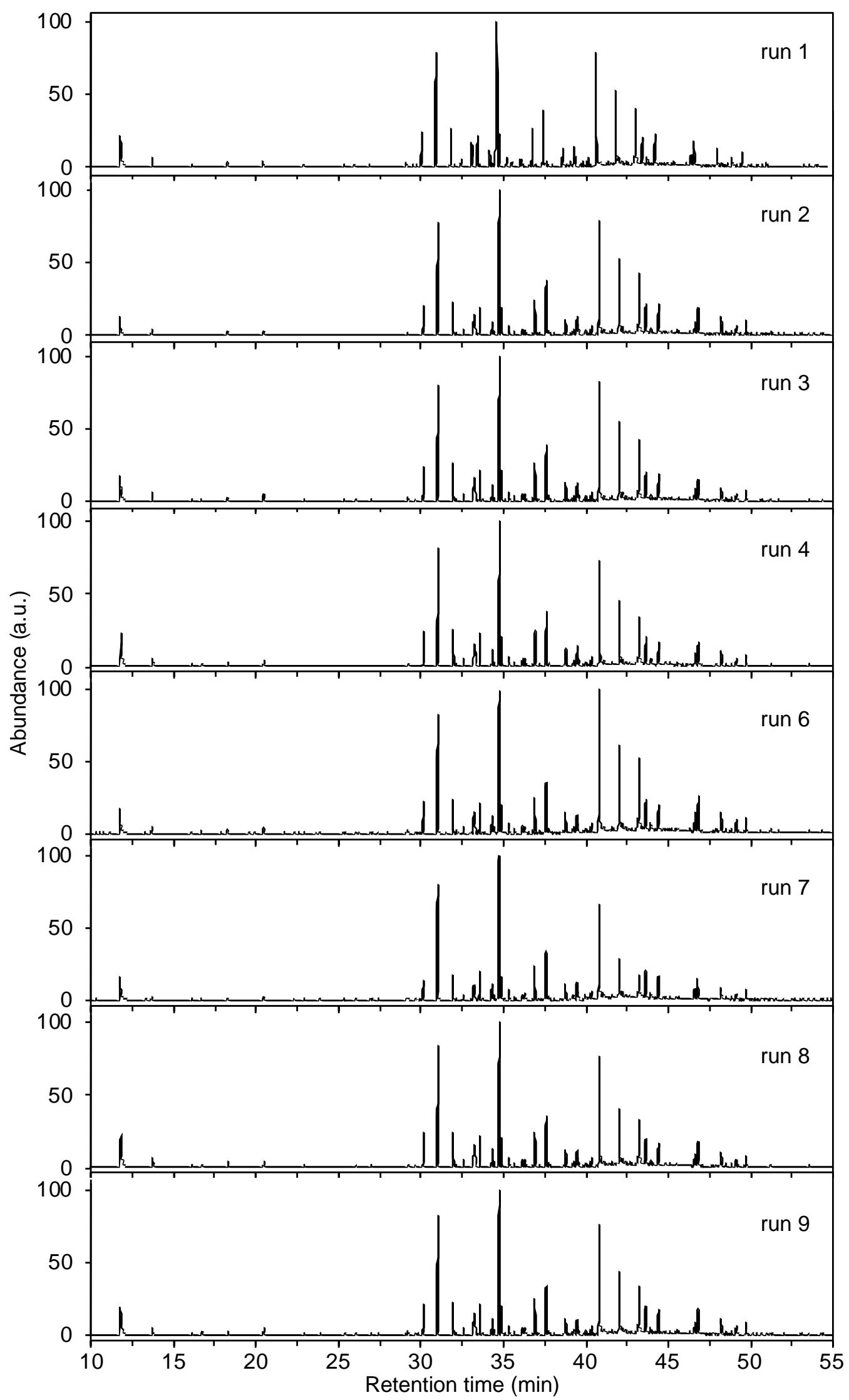

Figure S5. Total ion chromatograms of LOs in runs of 1-9. 
Table S1. List of compounds identified by GC/MS of LO in the first run.

\begin{tabular}{|c|c|c|c|c|}
\hline Number & Time (min) & Compound/ Formula & $\mathrm{Mw}$ & Area $\%$ \\
\hline 1 & 5.465 & methanol/ $\mathrm{CH}_{4} \mathrm{O}$ & 32 & 3.81 \\
\hline 2 & 7.384 & iso-butyraldehyde/ $\mathrm{C}_{4} \mathrm{H}_{8} \mathrm{O}$ & 72 & 0.04 \\
\hline 3 & 7.494 & 1,1-dimethoxyethane/ $\mathrm{C}_{4} \mathrm{H}_{10} \mathrm{O}_{2}$ & 90 & 0.05 \\
\hline 4 & 8.264 & allyl alcohol/ $\mathrm{C}_{3} \mathrm{H}_{6} \mathrm{O}$ & 58 & 0.05 \\
\hline 5 & 8.569 & butenone/ $\mathrm{C}_{4} \mathrm{H}_{6} \mathrm{O}$ & 70 & 0.10 \\
\hline 6 & 8.689 & 2,3-butanedione/ $\mathrm{C}_{4} \mathrm{H}_{6} \mathrm{O}_{2}$ & 86 & 0.06 \\
\hline 7 & 8.744 & 2-butanone/ $\mathrm{C}_{4} \mathrm{H}_{8} \mathrm{O}$ & 72 & 0.18 \\
\hline 8 & 9.009 & methyl propionate/ $\mathrm{C}_{4} \mathrm{H}_{8} \mathrm{O}_{2}$ & 88 & 0.02 \\
\hline 9 & 9.569 & benzene/ $\mathrm{C}_{6} \mathrm{H}_{6}$ & 86 & 0.12 \\
\hline 10 & 10.365 & pentanal/ $\mathrm{C}_{5} \mathrm{H}_{10} \mathrm{O}$ & 86 & 0.05 \\
\hline 11 & 10.835 & 2,5-dimethylfuran $/ \mathrm{C}_{6} \mathrm{H}_{8} \mathrm{O}$ & 96 & 0.04 \\
\hline 12 & 11.205 & crotonaldehyde/ $\mathrm{C}_{4} \mathrm{H}_{6} \mathrm{O}$ & 70 & 0.05 \\
\hline 13 & 11.785 & acetic acid $/ \mathrm{C}_{2} \mathrm{H}_{4} \mathrm{O}_{2}$ & 60 & 3.66 \\
\hline 14 & 13.386 & acetol/ $\mathrm{C}_{3} \mathrm{H}_{6} \mathrm{O}_{2}$ & 74 & 0.06 \\
\hline 15 & 13.751 & toluene/ $\mathrm{C}_{7} \mathrm{H}_{8}$ & 92 & 0.56 \\
\hline 16 & 15.017 & 3-penten-2-one/ $\mathrm{C}_{5} \mathrm{H}_{8} \mathrm{O}$ & 84 & 0.02 \\
\hline 17 & 16.172 & propanoic acid/ $\mathrm{C}_{3} \mathrm{H}_{6} \mathrm{O}_{2}$ & 94 & 0.16 \\
\hline 18 & 17.608 & cyclopentanone/ $\mathrm{C}_{5} \mathrm{H}_{8} \mathrm{O}$ & 84 & 0.02 \\
\hline 19 & 17.993 & $m$-xylene/ $\mathrm{C}_{8} \mathrm{H}_{10}$ & 106 & 0.03 \\
\hline 20 & 18.328 & $o$-xylene/ $\mathrm{C}_{8} \mathrm{H}_{10}$ & 106 & 0.31 \\
\hline 21 & 19.639 & $p$-xylene/ $\mathrm{C}_{8} \mathrm{H}_{10}$ & 106 & 0.04 \\
\hline 22 & 20.519 & furfural/ $\mathrm{C}_{5} \mathrm{H}_{4} \mathrm{O}_{2}$ & 96 & 0.45 \\
\hline 23 & 21.799 & anisole $/ \mathrm{C}_{7} \mathrm{H}_{8} \mathrm{O}$ & 108 & 0.03 \\
\hline 24 & 22.37 & 2-furanmethanol/ $\mathrm{C}_{5} \mathrm{H}_{6} \mathrm{O}_{2}$ & 98 & 0.06 \\
\hline 25 & 22.66 & 1,2,3-trimethylbenzene/ $\mathrm{C}_{9} \mathrm{H}_{12}$ & 120 & 0.04 \\
\hline 26 & 22.98 & 2-methyl-5-ethylfuran/ $\mathrm{C}_{6} \mathrm{H}_{8} \mathrm{O}$ & 110 & 0.11 \\
\hline 27 & 23.605 & 2-ethyl-5-methylfuran/ $\mathrm{C}_{7} \mathrm{H}_{10} \mathrm{O}$ & 110 & 0.02 \\
\hline 28 & 23.925 & mesitylene/ $\mathrm{C}_{9} \mathrm{H}_{12}$ & 120 & 0.05 \\
\hline 29 & 25.361 & 2-methylanisole $/ \mathrm{C}_{8} \mathrm{H}_{10} \mathrm{O}$ & 122 & 0.05 \\
\hline 30 & 25.446 & benzofuran $/ \mathrm{C}_{8} \mathrm{H}_{6} \mathrm{O}$ & 118 & 0.08 \\
\hline 31 & 26.131 & 3-methylanisole $/ \mathrm{C}_{8} \mathrm{H}_{10} \mathrm{O}$ & 122 & 0.15 \\
\hline 32 & 26.321 & 5-methylfurfural $/ \mathrm{C}_{6} \mathrm{H}_{6} \mathrm{O}_{2}$ & 110 & 0.05 \\
\hline 33 & 27.026 & 3-methyl-2-cyclopenten-1-one/ $\mathrm{C}_{6} \mathrm{H}_{8} \mathrm{O}$ & 96 & 0.08 \\
\hline 34 & 27.131 & 4-ethynyltoluene/ $\mathrm{C}_{9} \mathrm{H}_{8}$ & 116 & 0.04 \\
\hline 35 & 27.477 & $2(5 \mathrm{H})$-furanone $/ \mathrm{C}_{4} \mathrm{H}_{4} \mathrm{O}_{2}$ & 84 & 0.06 \\
\hline 36 & 28.037 & 5-methyl-2(5H)-furanone/ $\mathrm{C}_{5} \mathrm{H}_{6} \mathrm{O}_{2}$ & 98 & 0.03 \\
\hline 37 & 29.162 & 2-ethyl-6-methylphenol/ $\mathrm{C}_{9} \mathrm{H}_{12} \mathrm{O}$ & 136 & 0.05 \\
\hline 38 & 29.267 & 2,3-dimethylanisole/ $\mathrm{C}_{9} \mathrm{H}_{12} \mathrm{O}$ & 136 & 0.29 \\
\hline 39 & 29.723 & 7-methylbenzofuran/ $\mathrm{C}_{9} \mathrm{H}_{8} \mathrm{O}$ & 132 & 0.12 \\
\hline 40 & 29.873 & 2-methylbenzofuran/ $\mathrm{C}_{9} \mathrm{H}_{8} \mathrm{O}$ & 132 & 0.05 \\
\hline 41 & 30.008 & 3,5-dimethylanisole/ $\mathrm{C}_{9} \mathrm{H}_{12} \mathrm{O}$ & 136 & 0.06 \\
\hline 42 & 30.218 & phenol/ $\mathrm{C}_{6} \mathrm{H}_{6} \mathrm{O}$ & 94 & 2.28 \\
\hline 43 & 31.098 & guaiacol/ $\mathrm{C}_{7} \mathrm{H}_{8} \mathrm{O} 2$ & 124 & 10.93 \\
\hline 44 & 31.603 & 3-methyl-1H-indene/ $\mathrm{C}_{10} \mathrm{H}_{10}$ & 130 & 0.02 \\
\hline 45 & 32.013 & $o$-cresol/ $\mathrm{C}_{7} \mathrm{H}_{8} \mathrm{O}$ & 108 & 2.42 \\
\hline 46 & 32.239 & 1,2-dimethoxybenzene/ $\mathrm{C}_{8} \mathrm{H}_{10} \mathrm{O}_{2}$ & 138 & 0.12 \\
\hline 47 & 32.649 & 2,6-dimethylphenol/ $\mathrm{C}_{8} \mathrm{H}_{10} \mathrm{O}$ & 122 & 0.46 \\
\hline 48 & 33.279 & $p$-cresol/ $\mathrm{C}_{7} \mathrm{H}_{8} \mathrm{O}$ & 108 & 1.64 \\
\hline 49 & 33.344 & $m$-cresol $/ \mathrm{C}_{7} \mathrm{H}_{8} \mathrm{O}$ & 108 & 1.42 \\
\hline 50 & 33.644 & 2-methoxy-6-methylphenol/ $\mathrm{C}_{8} \mathrm{H}_{10} \mathrm{O}_{2}$ & 138 & 2.03 \\
\hline 51 & 33.819 & 4,7-dimethylbenzofuran/ $\mathrm{C}_{10} \mathrm{H}_{10} \mathrm{O}$ & 146 & 0.11 \\
\hline 52 & 34.389 & 2-methoxy-5-methylphenol/ $\mathrm{C}_{8} \mathrm{H}_{10} \mathrm{O}_{2}$ & 138 & 1.01 \\
\hline 53 & 34.519 & levoglucosenone/ $\mathrm{C}_{6} \mathrm{H}_{6} \mathrm{O}_{3}$ & 126 & 0.22 \\
\hline 54 & 34.83 & creosol/ $\mathrm{C}_{8} \mathrm{H}_{10} \mathrm{O}_{2}$ & 138 & 14.71 \\
\hline
\end{tabular}




\begin{tabular}{|c|c|c|c|c|}
\hline 55 & 34.965 & 2,5-dimethylphenol/ $\mathrm{C}_{8} \mathrm{H}_{10} \mathrm{O}$ & 122 & 2.06 \\
\hline 56 & 35.16 & 2-ethyl-6-methylphenol/ $\mathrm{C}_{9} \mathrm{H}_{12} \mathrm{O}$ & 136 & 0.06 \\
\hline 57 & 35.425 & 3,4-dimethoxytoluene/ $\mathrm{C}_{9} \mathrm{H}_{12} \mathrm{O}_{2}$ & 152 & 0.48 \\
\hline 58 & 35.515 & 4,6-dimethyl-2- $\alpha$-pyrone/ $\mathrm{C}_{7} \mathrm{H}_{8} \mathrm{O}_{2}$ & 124 & 0.04 \\
\hline 59 & 35.735 & 2,4,6-trimethylphenol/ $\mathrm{C}_{9} \mathrm{H}_{12} \mathrm{O}$ & 136 & 0.27 \\
\hline 60 & 36.23 & 3,5-dimethylphenol/ $\mathrm{C}_{8} \mathrm{H}_{10} \mathrm{O}$ & 122 & 0.43 \\
\hline 61 & 36.36 & 4-ethylphenol/ $\mathrm{C}_{8} \mathrm{H}_{10} \mathrm{O}$ & 122 & 0.48 \\
\hline 62 & 36.44 & 3-ethylphenol/ $\mathrm{C}_{8} \mathrm{H}_{10} \mathrm{O}$ & 122 & 0.08 \\
\hline 63 & 36.64 & 6-ethyl-2-methyl-decane/ $\mathrm{C}_{13} \mathrm{H}_{28}$ & 184 & 0.01 \\
\hline 64 & 36.845 & estragole/ $\mathrm{C}_{10} \mathrm{H}_{12} \mathrm{O}$ & 148 & 0.19 \\
\hline 65 & 36.99 & 3,4-dimethoxytoluene/ $\mathrm{C}_{9} \mathrm{H}_{12} \mathrm{O}_{2}$ & 152 & 2.48 \\
\hline 66 & 37.181 & 1-ethylidene-1H-indene/ $\mathrm{C}_{11} \mathrm{H}_{10}$ & 142 & 0.07 \\
\hline 67 & 37.251 & 3,4-dimethylphenol/ $\mathrm{C}_{8} \mathrm{H}_{10} \mathrm{O}$ & 122 & 0.13 \\
\hline 68 & 37.396 & 2-isopropylphenol/ $\mathrm{C}_{9} \mathrm{H}_{12} \mathrm{O}$ & 136 & 0.04 \\
\hline 69 & 37.671 & 4-ethylguaiacol/ $\mathrm{C}_{9} \mathrm{H}_{12} \mathrm{O}_{2}$ & 152 & 3.80 \\
\hline 70 & 37.856 & 2-ethyl-5-methylphenol/ $\mathrm{C}_{9} \mathrm{H}_{12} \mathrm{O}$ & 136 & 0.34 \\
\hline 71 & 37.951 & 3-ethyl-5-methylphenol/ $\mathrm{C}_{9} \mathrm{H}_{12} \mathrm{O}$ & 136 & 0.07 \\
\hline 72 & 38.186 & 4-ethyl-1,2-dimethoxybenzene/ $\mathrm{C}_{10} \mathrm{H}_{14} \mathrm{O}_{2}$ & 166 & 0.07 \\
\hline 73 & 38.561 & 2,3,4,6-tetramethylphenol/ $\mathrm{C}_{10} \mathrm{H}_{14} \mathrm{O}$ & 150 & 0.05 \\
\hline 74 & 38.611 & 2-(1-methylethylidene)-cyclohexanone/ $\mathrm{C}_{9} \mathrm{H}_{14} \mathrm{O}$ & 138 & 0.04 \\
\hline 75 & 38.751 & 3,4,5-trimethylphenol/ $\mathrm{C}_{9} \mathrm{H}_{12} \mathrm{O}_{2}$ & 136 & 0.10 \\
\hline 76 & 38.856 & 2,5-dimethoxytoluene/ $\mathrm{C}_{9} \mathrm{H}_{12} \mathrm{O}_{2}$ & 152 & 1.19 \\
\hline 77 & 38.931 & 2,3,5-trimethylphenol/ $\mathrm{C}_{9} \mathrm{H}_{12} \mathrm{O}$ & 136 & 0.11 \\
\hline 78 & 39.066 & 3-ethyl-5-methylphenol/ $\mathrm{C}_{9} \mathrm{H}_{12} \mathrm{O}$ & 136 & 0.05 \\
\hline 79 & 39.181 & 2,5-dimethoxyethylbenzene/ $\mathrm{C}_{10} \mathrm{H}_{14} \mathrm{O}_{2}$ & 166 & 0.06 \\
\hline 80 & 39.331 & 3-methoxy-2,4,5-trimethylphenol/ $\mathrm{C}_{10} \mathrm{H}_{14} \mathrm{O}_{2}$ & 166 & 0.40 \\
\hline 81 & 39.541 & 2-methoxy-4-vinylphenol/ $\mathrm{C}_{9} \mathrm{H}_{10} \mathrm{O}_{2}$ & 150 & 1.21 \\
\hline 82 & 39.616 & 2,5-diol-p-cymene/ $\mathrm{C}_{10} \mathrm{H}_{14} \mathrm{O}_{2}$ & 166 & 0.76 \\
\hline 83 & 39.952 & 2-isopropoxyphenol/ $\mathrm{C}_{9} \mathrm{H}_{12} \mathrm{O}_{2}$ & 152 & 0.08 \\
\hline 84 & 40.077 & 4-hydroxybenzylideneacetone/ $\mathrm{C}_{10} \mathrm{H}_{10} \mathrm{O}_{2}$ & 162 & 0.35 \\
\hline 85 & 40.197 & 4-ethenyl-1,2-dimethoxybenzene/ $\mathrm{C}_{10} \mathrm{H}_{12} \mathrm{O}_{2}$ & 164 & 0.09 \\
\hline 86 & 40.242 & 2,7-dimethyl-3(2H)-benzofuranone/ $\mathrm{C}_{10} \mathrm{H}_{10} \mathrm{O}_{2}$ & 162 & 0.05 \\
\hline 87 & 40.332 & eugenol/ $\mathrm{C}_{10} \mathrm{H}_{12} \mathrm{O}_{2}$ & 164 & 0.32 \\
\hline 88 & 40.437 & 2-methoxy-4-propylphenol/ $\mathrm{C}_{10} \mathrm{H}_{14} \mathrm{O}_{2}$ & 166 & 0.52 \\
\hline 89 & 40.587 & 2-methyl-6-propylphenol/ $\mathrm{C}_{10} \mathrm{H}_{14} \mathrm{O}$ & 150 & 0.05 \\
\hline 90 & 40.907 & catechol/ $\mathrm{C}_{6} \mathrm{H}_{6} \mathrm{O}_{2}$ & 110 & 9.53 \\
\hline 91 & 41.177 & syringol/ $\mathrm{C}_{9} \mathrm{H}_{12} \mathrm{O}_{4}$ & 184 & 0.46 \\
\hline 92 & 41.527 & dihydrojasmone/ $\mathrm{C}_{11} \mathrm{H}_{18} \mathrm{O}$ & 166 & 0.09 \\
\hline 93 & 41.662 & 2-methoxy-5-[(E)-1-propenyl]-phenol/ $\mathrm{C}_{10} \mathrm{H}_{12} \mathrm{O}_{2}$ & 164 & 0.24 \\
\hline 94 & 42.102 & 3-methylcatechol $/ \mathrm{C}_{7} \mathrm{H}_{8} \mathrm{O}_{2}$ & 124 & 5.23 \\
\hline 95 & 42.288 & 3-methoxy-5-methylphenol/ $\mathrm{C}_{8} \mathrm{H}_{10} \mathrm{O}_{2}$ & 138 & 0.52 \\
\hline 96 & 42.733 & 3-hydroxybenzenemethanol / $\mathrm{C}_{7} \mathrm{H}_{8} \mathrm{O}_{2}$ & 124 & 0.25 \\
\hline 97 & 42.853 & 2-methyl-1,4-benzenediol/ $\mathrm{C}_{7} \mathrm{H}_{8} \mathrm{O}_{2}$ & 124 & 0.05 \\
\hline 98 & 43.323 & 4-methylcatechol/ $\mathrm{C}_{7} \mathrm{H}_{8} \mathrm{O}_{2}$ & 124 & 5.08 \\
\hline 99 & 43.718 & $c i s$-isoeugenol/ $\mathrm{C}_{10} \mathrm{H}_{12} \mathrm{O}_{2}$ & 164 & 1.78 \\
\hline 100 & 44.018 & isoeugenol/ $\mathrm{C}_{10} \mathrm{H}_{12} \mathrm{O}_{2}$ & 164 & 0.50 \\
\hline 101 & 44.508 & vanillin/ $\mathrm{C}_{8} \mathrm{H}_{8} \mathrm{O}_{3}$ & 152 & 2.41 \\
\hline 102 & 44.814 & 5-methoxy-2,3-dimethylphenol/ $\mathrm{C}_{9} \mathrm{H}_{12} \mathrm{O}_{2}$ & 152 & 0.09 \\
\hline 103 & 44.904 & 2,5-dimethyl-1,3-benzenediol/ $\mathrm{C}_{8} \mathrm{H}_{10} \mathrm{O}_{2}$ & 138 & 0.19 \\
\hline 104 & 45.219 & 4-hydroxybenzylideneacetone/ $\mathrm{C}_{10} \mathrm{H}_{10} \mathrm{O}_{2}$ & 162 & 0.19 \\
\hline 105 & 45.519 & 4-ethyl-1,3-benzenediol/ $\mathrm{C}_{8} \mathrm{H}_{10} \mathrm{O}_{2}$ & 138 & 0.10 \\
\hline 106 & 45.609 & $\begin{array}{l}\text { 2-methoxy-4-methyl-6-[(1E)-1-propenyl]-phenol/ } \\
\qquad \mathrm{C}_{11} \mathrm{H}_{14} \mathrm{O}_{2}\end{array}$ & 178 & 0.21 \\
\hline 107 & 45.709 & $\begin{array}{l}\text { 3,3,4,7-tetramethyl-2-benzofuran-1(3H)-one/ } \\
\qquad \mathrm{C}_{12} \mathrm{H}_{14} \mathrm{O}_{2}\end{array}$ & 190 & 0.10 \\
\hline 108 & 45.794 & 4,5-dimethyl-1,3-benzenediol/ $\mathrm{C}_{8} \mathrm{H}_{10} \mathrm{O}_{2}$ & 138 & 0.07 \\
\hline 109 & 46.429 & 4-(3-aminobutyl)-2-methoxyphenol/ $\mathrm{C}_{11} \mathrm{H}_{17} \mathrm{NO}_{2}$ & 195 & 0.18 \\
\hline
\end{tabular}




\begin{tabular}{|c|c|c|c|c|}
\hline 110 & 46.704 & methyl vanillate/ $\mathrm{C}_{9} \mathrm{H}_{10} \mathrm{O}_{4}$ & 182 & 0.87 \\
\hline 111 & 46.904 & acetovanillone/ $\mathrm{C}_{9} \mathrm{H}_{10} \mathrm{O}_{3}$ & 166 & 1.78 \\
\hline 112 & 46.974 & 5-methoxy-2,3,4-trimethylphenol/ $\mathrm{C}_{10} \mathrm{H}_{14} \mathrm{O}_{2}$ & 166 & 0.06 \\
\hline 113 & 47.114 & $\begin{array}{l}\text { 3,3,4,6-tetramethyl-1-benzofuran-2(3H)-one/ } \\
\qquad \mathrm{C}_{12} \mathrm{H}_{14} \mathrm{O}_{2}\end{array}$ & 190 & 0.05 \\
\hline 114 & 47.18 & $\begin{array}{l}\text { 4-(2,6,6-trimethyl-1-cyclohexen-1-yl)-2-butanone/ } \\
\qquad \mathrm{C}_{13} \mathrm{H}_{22} \mathrm{O}\end{array}$ & 194 & 0.09 \\
\hline 115 & 47.43 & 4-(but-2-oxy)-benzaldehyde/ $\mathrm{C}_{11} \mathrm{H}_{14} \mathrm{O}_{2}$ & 178 & 0.07 \\
\hline 116 & 47.685 & methyl p-tolyl-sulfoxide/ $\mathrm{C}_{8} \mathrm{H}_{10} \mathrm{OS}$ & 154 & 0.06 \\
\hline 117 & 47.87 & 1-(2-methoxyphenyl)-3-buten-1-ol/ $\mathrm{C}_{11} \mathrm{H}_{14} \mathrm{O}_{2}$ & 178 & 0.05 \\
\hline 118 & 48.005 & 2,4-dimethoxyacetophenone/ $\mathrm{C}_{10} \mathrm{H}_{12} \mathrm{O}_{3}$ & 180 & 0.17 \\
\hline 119 & 48.31 & methyl vanillyl ketone/ $\mathrm{C}_{10} \mathrm{H}_{12} \mathrm{O}_{3}$ & 180 & 1.48 \\
\hline 120 & 48.455 & 4-methoxy-2-[(3E)-3-penten-2-yl]-phenol/ $\mathrm{C}_{12} \mathrm{H}_{16} \mathrm{O}_{2}$ & 192 & 0.03 \\
\hline 121 & 48.575 & $\begin{array}{l}\text { methyl (4-hydroxy-3-methoxyphenyl)-acetate/ } \\
\qquad \mathrm{C}_{10} \mathrm{H}_{12} \mathrm{O}_{4}\end{array}$ & 196 & 0.25 \\
\hline 122 & 48.94 & 1-naphthalenol/ $\mathrm{C}_{11} \mathrm{H}_{16} \mathrm{O}_{2}$ & 144 & 0.22 \\
\hline 123 & 49.22 & $\begin{array}{l}\text { 1-(2-hydroxy-4-methoxyphenyl)-1-propanone/ } \\
\qquad \mathrm{C}_{10} \mathrm{H}_{12} \mathrm{O}_{3}\end{array}$ & 180 & 0.66 \\
\hline 124 & 49.33 & 3-methoxy-2,5,6-trimethylphenol/ $\mathrm{C}_{10} \mathrm{H}_{14} \mathrm{O}_{2}$ & 166 & 0.11 \\
\hline 125 & 49.49 & 3-phenylfuran/ $\mathrm{C}_{10} \mathrm{H}_{8} \mathrm{O}$ & 144 & 0.05 \\
\hline 126 & 49.731 & 4-butyl-1,2-dimethoxybenzene/ $\mathrm{C}_{12} \mathrm{H}_{18} \mathrm{O}_{2}$ & 194 & 0.09 \\
\hline 127 & 49.836 & butyrovanillone/ $\mathrm{C}_{11} \mathrm{H}_{14} \mathrm{O}_{3}$ & 194 & 0.93 \\
\hline 128 & 49.971 & $\begin{array}{l}\text { 5-hydroxy-6-methoxy-1-benzofuran-3(2H)-one/ } \\
\qquad \mathrm{C}_{9} \mathrm{H}_{8} \mathrm{O}_{4}\end{array}$ & 180 & 0.03 \\
\hline 129 & 50.246 & 2-ethyl-4,5-dimethylphenol/ $\mathrm{C}_{10} \mathrm{H}_{14} \mathrm{O}$ & 150 & 0.07 \\
\hline 130 & 50.541 & $\begin{array}{l}\text { 1,2-dimethoxy-4-(1-methoxyvinyl)-benzene/ } \\
\qquad \mathrm{C}_{11} \mathrm{H}_{14} \mathrm{O}_{3}\end{array}$ & 194 & 0.05 \\
\hline 131 & 50.716 & 2-tert-butyl-1,4-dimethoxybenzene/ $\mathrm{C}_{12} \mathrm{H}_{18} \mathrm{O}_{2}$ & 194 & 0.06 \\
\hline 132 & 51.086 & 1-(3,4-dimethoxyphenyl)-propan-1-one/ $\mathrm{C}_{11} \mathrm{H}_{14} \mathrm{O}_{3}$ & 194 & 0.03 \\
\hline 133 & 51.231 & 3-acetyl-2-methoxybenzo[b]furan/ $\mathrm{C}_{11} \mathrm{H}_{10} \mathrm{O}_{3}$ & 190 & 0.01 \\
\hline 134 & 51.321 & 3-methoxy-2-naphthalenol/ $\mathrm{C}_{11} \mathrm{H}_{10} \mathrm{O}_{2}$ & 174 & 0.17 \\
\hline 135 & 51.401 & butyrovanillone/ $\mathrm{C}_{11} \mathrm{H}_{14} \mathrm{O}_{3}$ & 194 & 0.04 \\
\hline 136 & 51.496 & $\begin{array}{l}\text { 4-(3-hydroxy-2-methoxyphenyl)-2-butanone/ } \\
\qquad \mathrm{C}_{11} \mathrm{H}_{14} \mathrm{O}_{3}\end{array}$ & 194 & 0.04 \\
\hline 137 & 51.611 & $\begin{array}{l}\text { 7-hydroxy-6-methoxy-1-benzofuran-3(2H)-one/ } \\
\qquad \mathrm{C}_{9} \mathrm{H}_{8} \mathrm{O}_{4}\end{array}$ & 180 & 0.03 \\
\hline
\end{tabular}


Table S2. Yield of major compounds of LOs quantified in runs of $1-9$ (wt $\%$-dry lignin).

\begin{tabular}{ccccccccc}
\hline compound & run 1 & run 2 & run 3 & run 4 & run 6 & run 7 & run 8 & run 9 \\
\hline methanol & 0.19 & 0.21 & 0.20 & 0.19 & 0.21 & 0.22 & 0.21 & 0.22 \\
acetic acid & 0.86 & 0.95 & 0.77 & 1.10 & 0.95 & 1.39 & 1.29 & 1.50 \\
toluene & 0.03 & 0.03 & 0.03 & 0.04 & 0.03 & 0.03 & 0.05 & 0.03 \\
xylene & 0.02 & 0.02 & 0.03 & 0.02 & 0.02 & 0.02 & 0.03 & 0.02 \\
phenol & 0.16 & 0.16 & 0.17 & 0.21 & 0.19 & 0.19 & 0.24 & 0.25 \\
guaiacol & 0.52 & 0.56 & 0.57 & 0.73 & 0.64 & 0.75 & 0.81 & 0.92 \\
creosol & 0.68 & 0.70 & 0.71 & 0.90 & 0.76 & 0.70 & 0.97 & 1.13 \\
cresol & 0.30 & 0.29 & 0.34 & 0.41 & 0.35 & 0.35 & 0.46 & 0.47 \\
xylenol & 0.18 & 0.18 & 0.18 & 0.23 & 0.21 & 0.22 & 0.26 & 0.29 \\
4-ethylphenol & 0.01 & 0.01 & 0.01 & 0.01 & 0.01 & 0.01 & 0.01 & 0.02 \\
4-ethylguaiacol & 0.25 & 0.27 & 0.25 & 0.31 & 0.28 & 0.35 & 0.34 & 0.40 \\
catechol & 1.20 & 1.13 & 1.27 & 1.43 & 1.80 & 1.95 & 1.95 & 1.97 \\
syringol & 0.05 & 0.03 & 0.03 & 0.06 & 0.07 & 0.06 & 0.08 & 0.07 \\
3-methylcatechol & 0.74 & 0.71 & 1.18 & 1.17 & 1.51 & 1.53 & 1.57 & 1.12 \\
4-methylcatechol & 1.29 & 1.50 & 2.01 & 2.15 & 3.06 & 2.41 & 2.35 & 2.58 \\
vanillin & 0.24 & 0.22 & 0.19 & 0.23 & 0.22 & 0.25 & 0.24 & 0.34 \\
apocynin & 0.14 & 0.16 & 0.14 & 0.18 & 0.26 & 0.24 & 0.21 & 0.26 \\
total & 6.97 & 7.25 & 8.17 & 9.50 & 10.72 & 10.87 & 11.22 & 11.78 \\
\hline
\end{tabular}

